\title{
Nanostructured hybrid materials for bone-tooth unit regeneration
}

\author{
Sabine Kuchler-Bopp ${ }^{1,2}$, Thibault Bécavin ${ }^{1,2,3}$, Tunay Kökten ${ }^{1,2}$, Florence Fioretti ${ }^{1,2}$, \\ Etienne Deveaux ${ }^{3}$, Nadia Benkirane-Jessel ${ }^{1,2^{*}}$, Laetitia Keller $^{1,2^{*}}$ \\ ${ }^{1}$ Institut National de la Santé et de la Recherche Médicale (INSERM), UMR 1109, Osteoarticular and Dental Regenerative \\ Nanomedicine, Faculté de Médecine, Strasbourg, France; *Corresponding Authors: nadia.jessel@inserm.fr, laetitia.keller@inserm.fr \\ ${ }^{2}$ Université de Strasbourg, Faculté de Chirurgie Dentaire, 1 Place de l'Hôpital, Strasbourg, France \\ ${ }^{3}$ Université Lille Nord de France, Faculté de Chirurgie Dentaire, Lille, France
}

Received 19 April 2013; revised 22 May 2013; accepted 16 June 2013

Copyright (C) 2013 Sabine Kuchler-Bopp et al. This is an open access article distributed under the Creative Commons Attribution License, which permits unrestricted use, distribution, and reproduction in any medium, provided the original work is properly cited.

\section{ABSTRACT}

As a part of regenerative medicine, biomaterials are largely used in this field of nanotechnology and tissue engineering research. We have recently developed a new scaffold using electrospun nanofibers of Poly ( $\varepsilon$-caprolactone), PCL which is able to mimic the collagen extracellular matrix of cells. The aim of this study was to engineer a biological and implantable structure leading the regeneration of the tooth-bone unit. For this aim, we have cultured mouse osteoblasts embedded in a collagen gel on the nanofibrous membrane and coupled this structure with an embryonic dental germ before implantation. To follow bone and tooth regeneration, we have performed RT-PCR, histology and immunofluorescence analysis. We showed here that this leaving implantable structure represents an accurate strategy for bone-tooth unit regeneration. We report here the first demonstration of bone-tooth unit regeneration by using a strategy based on a synthetic nanostructured membrane. This electrospun membrane is manufactured by using an FDA approved polymer, PCL and functionalized with osteoblasts before incorporation of the tooth germs at ED14 (the first lower molars) to generate bone-tooth unit in vivo after implantation in mice. Our technology represents an excellent platform on which other sophisticated products could be based.

Keywords: Bone; Nanostructured Material; Osteoblast; Tissue Engineering; Tooth

\section{INTRODUCTION}

Biomaterials play central roles in modern strategies in regenerative medicine and tissue engineering as designable biophysical and biochemical environment that direct cellular behavior and function. The fibrillar collagens are the most abundant natural polymers in the body and are found throughout the interstitial spaces with an essential function to impart structural integrity and strength to tissues. In the native tissues, the structural extracellular matrix $(\mathrm{ECM})$ proteins range in diameter from 50 to 500 nm. In order to create scaffolds or ECM analogues, which are truly biomimicking at this scale, one must employ nanotechnology. Recent advances in nanotechnology have led to a variety of approaches for the development of engineered ECM analogues. To date, three processing techniques (self-assembly, phase separation, and electrospinning) have evolved to allow the fabrication of nanofibrous scaffolds. With these advances, the longawaited and much anticipated construction of a truly "biomimicking" or "ideal" tissue engineered environment, or scaffold, for a variety of tissues is now highly feasible.

The intricate fibrillar architecture of natural ECM components has inspired several researchers to produce materials with similar structure. Upon fibers that are tens of microns in diameter, cells seem to respond as though to a 2-D substrate, acquiring an unnatural flat shape, leading to a nonphysiological, asymmetrical occupation of adhesion receptors; notwithstanding, such matrices have already shown remarkable success in tissue engineering applications, such as in the reconstruction of a dog urinary bladder [1] or as scaffolds for neural stem cells to facilitate regeneration after brain injury in a mouse stroke model [2]. Polymer processing technologies such as electrospinning [3] allow fiber formation 
down to the $10 \mathrm{~nm}$ scale. One difficulty in nanofiber technology is placing cells within a nanofibrillar structure with pore spaces much smaller than a cellular diameter; somehow the network must be formed in situ, around the cells, without cellular damage.

Tooth organ engineering is an area of regenerative medicine. One methodology of this field of research is based on biomimetic. It tries to replace tooth organ by mimic epithelial-mesenchymal interaction occurring during tooth organ development [4]. Today, to build an intact and biological tooth-bone unit and regenerate a functional anchoring system (root, peridental, ligament, alveolar bone) remains a major challenge in tooth organ engineering. Indeed, a correct anchoring system is needed for the complete tooth functionality, for avoiding tooth ankylosis and to prevent bone damage after tooth loss. It is expected that bioengineering technology will be developed for the reconstruction of fully functional organs in vitro through the precise arrangement of several different cell species.

Our strategy is based on an active and cellularized hydrogel and nanofibers as a matrix. In this study, we have used electrospun nanofibers of Poly ( $\varepsilon$-caprolactone) (PCL). PCL is degraded by hydrolysis of its ester linkages under physiological conditions (such as in the human body) and has therefore received a great deal of attention for use as an implantable biomaterial. In particular it is especially interesting for the preparation of long-term implantable devices, owing to its degradation, which is even slower than that of polylactide. PCL is a Food and Drug Administration (FDA) approved material that is used in the human body as (for example) a drug delivery device, suture (sold under the brand name Monocryl or generically), or adhesion barrier. We report here the first demonstration that we are able to regenerate bone-tooth unit by using a FDA approved electrospun membrane coated by mixed osteoblasts/collagen.

\section{MATERIALS AND METHODS}

\subsection{Chemicals and Electrospinning}

PCL, analytical grade, was purchased from Sigma Aldrich. PCL was dissolved in a mixture of dichloromethane/dimethylformamide (DCM/DMF $50 / 50 \mathrm{v} / \mathrm{v})$ at $15 \%$ $\mathrm{wt} / \mathrm{v}$ and was stirred overnight before use. Rat-tail type I collagen was purchased from Institut de Biotechnologies Jacques Boy. A homemade standard electrospinning setup was used to fabricate the PCL scaffolds. The PCL solution was poured into a $5 \mathrm{ml}$ syringe and ejected through a needle with a diameter of $0.5 \mathrm{~mm}$ at a flow rate of $1.2 \mathrm{ml} / \mathrm{h}$, thanks to a programmable pump (Harvard Apparatus). A high-voltage power supply (SPELLMAN, SL30P10) was used to set $15 \mathrm{kV}$ at the needle. Aluminum foils $\left(20 \times 20 \mathrm{~cm}^{2}\right)$, connected to the ground at a distance from the needle of $17 \mathrm{~cm}$, were used to collect the electrospun PCL scaffold.

\subsection{SEM Observation}

For morphological study, the PCL scaffolds were gold-coated (Edwards Sputter Coater) and observed with a Philips XL-30 ESEM scanning electron microscope in conventional mode (high vacuum) with a ThornleyEverhart secondary electron detector.

\subsection{Cells Culture}

Mice primary osteoblasts were obtain from parietal bone, cut in small pieces, washed in PBS and treated for $40 \mathrm{~min}$ at $37^{\circ} \mathrm{C}$ with PBS containing collagenase (50 $\mu \mathrm{g} / \mathrm{ml})$ and fungizone $(5 \mu \mathrm{g} / \mathrm{ml})$. After washing in PBS, bone pieces were cultured in Dulbecco's modified Eagle's medium $\left(\mathrm{D}-\mathrm{MEM}^{\mathbb{B}}\right.$ ) containing $50 \mathrm{U} / \mathrm{ml}$ penicillin, $50 \mu \mathrm{g} / \mathrm{ml}$ streptomycin, $5 \mu \mathrm{g} / \mathrm{ml}$ fungizone, $1 \%$ sodium pyruvate, $0.1 \%$ ascorbic acid and 10\% FBS (Life Technologies, Paisley, UK). The cultures were incubated at $37^{\circ} \mathrm{C}$ in a humidified atmosphere of $5 \% \mathrm{CO}_{2}$. After 4 days the medium was changed. The generated osteoblasts were cultured in $75 \mathrm{~cm}^{2}$ flasks and medium was changed every 3 days.

\subsection{Immunocytochemistry and Osteoblasts Mineralization Staining}

Cells were cultured for 14 days on electrospun PCL membrane, fixed with paraformaldehyde (PFA) 4\% during $1 \mathrm{~h}$, permeabilized with $0.1 \%$ PBS-Triton X-100 for $1 \mathrm{~h}$, saturated with BSA $0.1 \%$ and incubated for $20 \mathrm{~min}$ with Alexa Fluor 546-conjugated phalloidin (Molecular Probes) for F-actin labeling and 5 min with $200 \mathrm{nM}$ DAPI (Sigma) for nuclear staining. After saturation, cells were also incubated with the primary antibodies: Osteocalcin, Osteonectin, Osteopontin and Collagen I. After rinsing with PBS, cells were incubated with secondary antibodies; anti-goat alexa fluor 488 for Osteocalcin and Osteonectin and anti-rabbit alexa fluor 488 for Collagen I and Osteopontin. For hematoxylin staining, cells were fixed with PFA and then stained with hematoxylin $10 \%$ $(\mathrm{w} / \mathrm{v})$ for $15 \mathrm{~min}$, rinsed with water and observed. For red alizarin staining, cells were fixed with ethanol $70 \%$ and stained with red alizarin $1 \%(\mathrm{w} / \mathrm{v})$, dried and observed.

\subsection{RNA Isolation and RT-PCR Analysis}

RT-PCR was performed on osteoblasts cultured for 7 and 14 days. Total RNA was isolated by affinity chromatography using the RNeasy ${ }^{\circledR}$ Minikit (Qiagen Inc., Hilden, Germany) and reverse-transcribed with oligo (dT) 12 and Superscript III (Invitrogen), according to the manufacturer protocols. In each experiment $1 \mu \mathrm{g}$ of total 
RNA was used for RT-PCR amplification was carried out with the Go Taq Hot Start kit (Promega, France) according to the manufacturer's instructions, using the specific primers (Table 1). PCR conditions were as followed: initial denaturation of $3 \mathrm{~min}$ at $94^{\circ} \mathrm{C}$ followed by 35 cycles at $94^{\circ} \mathrm{C}$ for $1 \mathrm{~min}$, annealing for $1 \mathrm{~min}$ at $58^{\circ} \mathrm{C}$ and elongation for $1 \mathrm{~min}$ at $72^{\circ} \mathrm{C}$, with a final extension of $10 \mathrm{~min}$ at $72^{\circ} \mathrm{C}$. PCR products were separated by electrophoresis on $1.5 \%$ agarose gels.

\subsection{Molar ED14 Culture}

The first lower molars were dissected from ICR mouse (Charles River Laboratories), embryos at Embryonic Day (ED) 14. All procedures were in compliance with the recommendations of the European Economic Community (86/609/CEE) on use and care of laboratory animals. Molars have been cultured for 5 days on the electrospun PCL membrane and on a semi-solid medium as previously described $[5,6]$.

\subsection{Implant Preparation and in Vivo Implantation}

For the Collagen preparation, $3 \mathrm{ml}$ of Rat Tail Type I Collagen (Institut de Biotechnologies Jacques Boy) were mixed with $5.5 \mathrm{ml}$ of medium containing $10 \%$ FBS, 0.5 $\mathrm{ml}$ of a $0.1 \mathrm{M} \mathrm{NaOH}$ and $1 \mathrm{ml}$ of osteoblasts suspension at $2 \times 10^{5}$ cells $/ \mathrm{ml}$. $0.1 \mathrm{ml}$ of osteoblasts suspension/ collagen preparation were deposited on the top of the electrospun PCL membrane. After adding the ED14 tooth germs, the construct was incubated at $37^{\circ} \mathrm{C}$ for $30 \mathrm{~min}$ before implantation. For in vitro analysis, the osteoblasts were cultured for 7 and 14 days on electrospun PCL membrane. For in vivo analysis, the samples were implanted between skin and muscles behind the ears in mice ( 8 weeks old ICR) for 2 weeks. For histology, samples were fixed in Bouin-Hollande, embedded in paraffin and $5 \mu \mathrm{m}$ serial sections were stained with Mallory. Implanted samples were demineralized in $15 \%$ EDTA before embedding in paraffin. For immunofluorescence, implants were embedded in Tissue-Tek and frozen at $-20^{\circ} \mathrm{C}$. Sections $(10 \mu \mathrm{m})$ were stained with anti-CD31 (BD Pharmingen) for the detection of vascular endothelial cells, and with Osteopontin for the detection of bone.
After washing with PBS, sections were incubated with secondary anti-rabbit antibodies conjugated to Alexa 594 and anti-rat antibodies conjugated to Alexa 488 (Molecular Probes, Invitrogen).

\section{RESULTS AND DISCUSSION}

\subsection{In Vitro Bone Induction Analysis}

For bone induction analysis, we have currently analyzed the biocompatibility of our electrospun PCL membrane after incubation of primary mice osteoblasts by SEM analysis (Figure 1). Moreover, our results indicated clearly that after 4 hours, the adhesion of cells growing on the surface of the membrane become comparable to the positive control including plastic support (data not shown).

We have also studied the behavior of mice osteoblasts growing on nanofibrous PCL membrane by histology (Figures 2(A)-(C)) and immunofluorescence (Figures 2(D)-(H)). After 14 days in vitro, these cells expressed some bone specific proteins: collagen I, Osteonectin, Osteocalcin and Osteopontin (Figures 2(E)-(H)). Furthermore we showed a nice mineralization by red alzarin in these cells after 14 days in vitro (Figure 2 (C)) and normal actin distribution (Figure 2 (D)). Gene expression of these molecules was also analyzed by RT-PCR (Figure 3) in 7 and 14 days cultured osteoblasts on the electrospun PCL membrane. Our results demonstrated clearly that the nanofibrous PCL membrane could be considered as a suitable scaffold for bone tissue engineering.

\subsection{In Vivo Analysis of the Engineering Tooth-Bone Unit}

Recently, there has been an increasing interest and awareness of the importance of the sub-tooth bone for its role in the pathogenic processes. It's necessary to carefully consider this structure in the treatment of tooth damage, in the evaluation of the results over time and in the determination of the patient prognosis. In fact, the conditions of teeth and its supporting bone are tightly coupled and should be viewed as a connected bone-tooth unit.

For the in vivo tooth-bone unit regeneration, we ana-

Table 1. Forward and reverse primers used for RT-PCR.

\begin{tabular}{ccc}
\hline Primers & Forward & Reverse \\
\hline GAPDH & CCATGGAGAAGGCCGGGG & CAAAGTTGTCATGGATGACC \\
Collagen1 & AATGGTGAGACGTGGAAACCCGAG & CGACTCCTACATCTTCTGAGTTTGG \\
Osteopontin & GACCATGAGATTGACAGTGATTTG & TGATGTTCCAGGCTGGCTTTG \\
Osteocalcin & GACAAAGCCTTCATGTCCAAGC & AAAGCCGAGCTGCCAGAGTTTG \\
Osteonectin & TGAGAATGAGAAGCGCCTGGA & AAGGGGGTAATGGGAGGGGTG \\
\hline
\end{tabular}




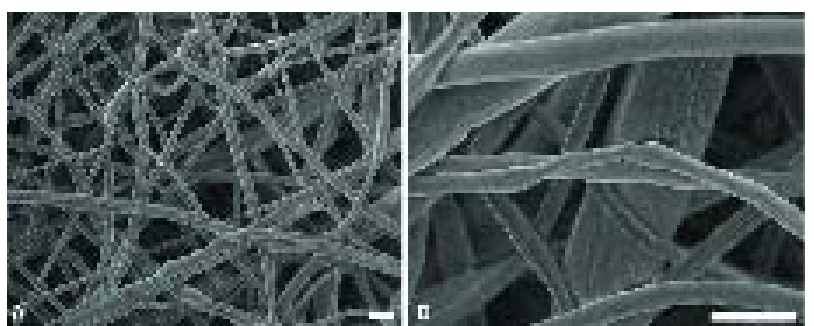

Figure 1. SEM observation of the nanofibrous PCL scaffolds (A, B). Bar $=1 \mu \mathrm{m}$.

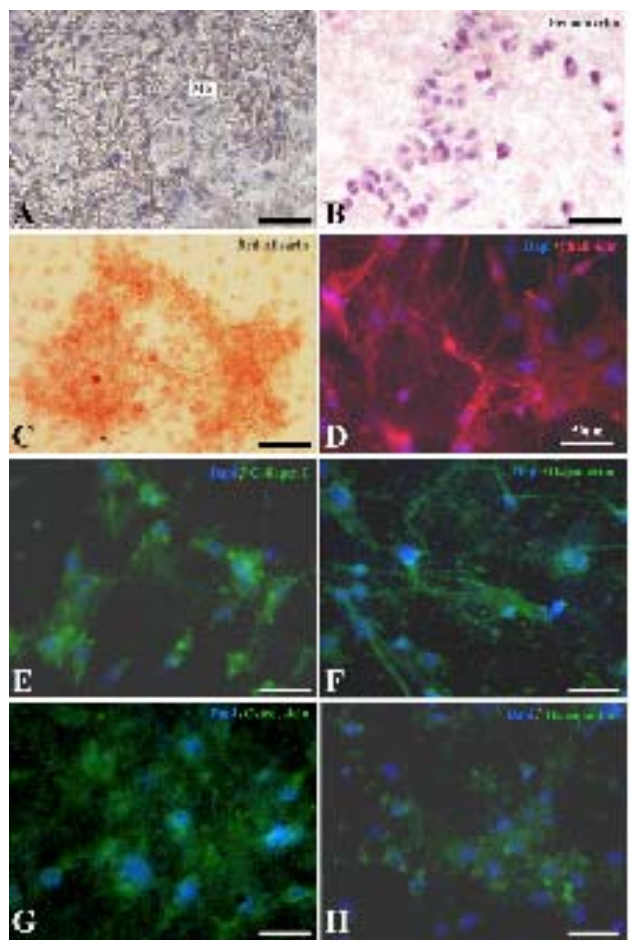

Figure 2. Behaviour of mice osteoblasts growing on PCL nanofibrous membrane during 14 days. Hematoxylin staining for histology ((A), (B)), red alizarin staining for mineralization $(\mathrm{C})$, alexa fluor 546-conjugated phalloidin for F-actin labeling (D) and immunofluorescence ((E)-(H)). Collagen I (E), Osteonectin (F), Osteocalcin (G), Osteopontin $(\mathrm{H})$. Bar $=50 \mu \mathrm{m}$.
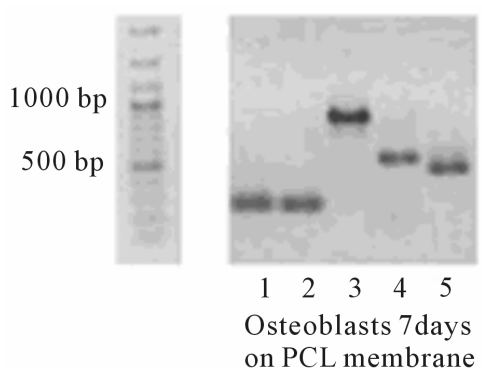

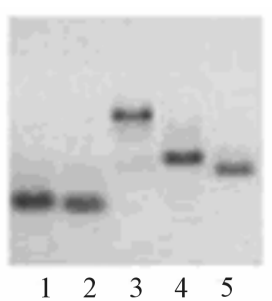

Osteoblasts 14days on PCL membrane
Figure 3. RT-PCR performed on mice osteoblasts cultured for 7 and 14 days on PCL membrane. The genes specifically observed were: Collagen I (1), Osteopontin (2), Osteocalcin (3), Osteonectin (4) and GADPH (5) for a control. lyzed the subcutaneous implantation of the ED14 first lower molars cultured during 5 days on the nanofibrous PCL membrane (Figure 4 (A)). We observed a correct development of the crown, including functional odontoblasts and ameloblasts secreting dentin and enamel respectively. Furthermore, the gradients of odontoblast differentiation were maintained in the root portion.

We then test the possibility to regenerate bone-tooth unit by using a hybrid nanostructured and living material. After in vivo implantation of the tooth germs at ED14, cultured 5 days on the membrane without adding osteoblasts (Figure 4 (A)), no bone induction was detected. Interestingly, by adding mixed osteoblasts/collagen as a coating of the membrane (nanostructured living membrane) and after incubation of the tooth germs at ED14 and implantation, we have shown bone induction (Figure 4 (B)). In these conditions, the developed tooth exhibited correct epithelial histogenesis (Figure 4 (B)) and allowed the functional differentiation of odontoblasts (Figure 4 (D)) and ameloblasts (Figures 4 (E)-(H)). Induced bone was observed around the tooth (Figures 4 (B), (F)-(H)) and especially near the root (Figures 4 (G) and (H)). Cementoblasts are in contact with the root dentin and begin to secrete cement (Figure $\mathbf{4}(\mathbf{H})$ ). At this stage of development, orientation of the future fibers of the peridental ligament can be yet observed (Figure $4(\mathbf{H})$ ). For more characterization of the induced bone-tooth unit growing on this nanofibers membrane, the blood vessels in the dental pulp and in the peripheral tissue were stained with an anti-CD31 antibody and the bone was visualized with anti-Osteopontin antibody (Figure 4 (I)). We then controlled that collagen had no effect on teeth development after implantation under skin (Figure 4 (C)). We attempt to implant ED14 molar with mixed murine osteoblasts/collagen without PCL membrane, with no results. Based on these results, we have reported here the first demonstration of a unique nanostructured material for bone-tooth unit regeneration in vivo. Thus, PCL nanofibrous membrane coated by mixed osteoblasts/collagen could represent a promising strategy of nanostructured living membrane for in vivo bone-tooth unit regeneration.

In the last years, more intentions are carrying on functionalization of different scaffold [3,7-18]. Multi layers film is one of the multiple technologies used to incorporate bioactives molecules [8-10]. Leading to a nanoarchitecture, these new generation of delivery molecules scaffold can provide the control of cell differentiation, inflammation $[11,12]$ by the diffusion of growth factors, nucleic acids $[13,15]$ in a controlled period of time and in a restricted localization. For example, the layer-by-layer technology can embedded active molecules into the multilayered films. Recently, we have designed an in vitro culture system based on an active film functionalized by 


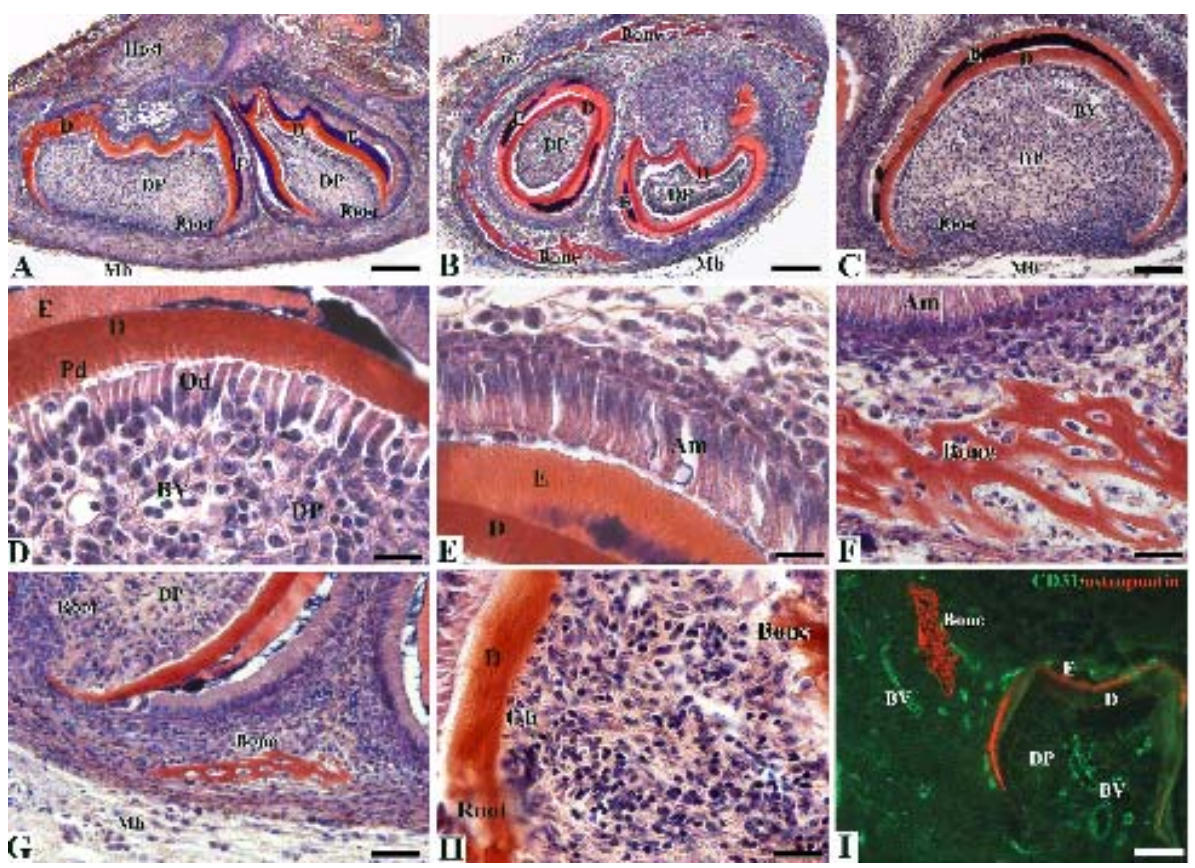

Figure 4. Bone-tooth unit regeneration in vivo. In vivo implantation for 2 weeks of ED14 first lower molars cultured 5 days on electrospun PCL membrane (A), on mixed murine osteoblasts/collagen coated electrospun PCL membrane ((B), (D)-(I)) or on PCL membrane in the presence of collagen $(\mathrm{C})$. The explants were stained with Mallory $((\mathrm{A})-(\mathrm{H}))$. The blood vessels in the dental pulp and in the peridental tissue were stained with the anti-CD31 antibody (green) and the regenerated bone with the anti-Osteopontin antibody (red) (I). Am: ameloblasts, BV: blood vessel, Cb: cementoblasts, D: dentin, DP: dental pulp, E: enamel, Mb: membrane, Od: odontoblasts, Pd: predentin. Bars $=100 \mu \mathrm{m}((\mathrm{A}),(\mathrm{B})), 50 \mu \mathrm{m}((\mathrm{C}),(\mathrm{I})), 25 \mu \mathrm{m}$ (G) and $12.5 \mu \mathrm{m}((\mathrm{D})-(\mathrm{F}),(\mathrm{H}))$.

BMP-4 and/or Noggin. Our results demonstrated clearly the possibility to control in situ apoptosis during tooth development mediated by both BMP-4 and Noggin incorporated into our active films [19]. We have also shown that BMP-2 functionalized PCL membrane was able to increase bone tissue regeneration after implantation [20]. To increase the regeneration of this complex bone-tooth unit, it could be relevant to combine these two strategies (membrane functionalization and tooth regeneration). Particular attention has to be done to understand the interaction between the new bone and the tooth root. To regenerate of real functional bone-tooth unit, the periodontal ligament formation must be also achieved.

\section{CONCLUSION}

With this strategy it should be possible to fabricate a combination cell-therapy implant capable of robust and durable tooth regeneration in large bone defects, when adding cells from patients become needed, to generate bone-tooth unit. We believe that our results make a significant contribution to the area of regenerative medicine and more precisely to bone and tooth related biomaterials. The concepts discovered here are applicable to a broad class of tissues and may serve to design sophisticated implants.

\section{ACKNOWLEDGEMENTS}

This work was supported by the project NEOTISSAGE from the "Agence Nationale de la Recherche, ANR" and "Alsace contre le Cancer", N. J. thanks the Faculté de Chirurgie Dentaire de Strasbourg for financial support. N. J. is indebted to CHU de Nancy, Hôpital Central, "Chirurgie Orthopédique et Traumatologie" (Contrat d'interface INSERM vers 1'hôpital). We are indebted to Hervé Gegout for his help and nice work on histology and Mathieu Erhardt for SEM.

\section{REFERENCES}

[1] Oberpenning, F., Meng, J., Yoo, J.J. and Atala, A. (1999) De novo reconstitution of a functional mammalian urinary bladder by tissue engineering. Nature Biotechnology, 17, 149-155. doi:10.1038/6146

[2] Park, K.I., Teng, Y.D. and Snyder, E.Y. (2002) The injured brain interacts reciprocally with neural stem cells supported by scaffolds to reconstitute lost tissue. Nature Biotechnology, 20, 1111-1117. doi:10.1038/nbt751

[3] Kenawy, el R., Layman, J.M., Watkins, J.R., Bowlin, G.L., Matthews, J.A., Simpson, D.G. and Wnek, G.E. (2003). Electrospinning of poly(ethylene-co-vinyl alcohol) fibers. 
Biomaterials, 24, 907-913. doi:10.1155/2011/201834

[4] Nait Lechguer, A., Couble, M.L., Labert, N., KuchlerBopp, S., Keller, L., Magloire, H., Bleicher, F. and Lesot, H. (2011) Cell differentiation and matrix organization in engineered teeth. Journal of Dental Research, 90, 583589. doi:10.1177/0022034510391796

[5] Nait Lechguer, A., Kuchler-Bopp, S., Hu, B., Haikel, Y. and Lesot, H. (2008) Vascularization of engineered teeth. Journal of Dental Research, 87, 1138-1143. doi: $10.1177 / 154405910808701216$

[6] Hu, B., Nadiri, A., Bopp-Kuchler, S., Perrin-Schmitt, F. and Lesot, H. (2005) Dental epithelial histomorphogenesis in vitro. Journal of Dental Research, 84, 521-525. doi:10.1177/154405910508400607

[7] Zhang, S. (2003) Fabrication of novel biomaterials through molecular self-assembly. Nature Biotechnology, 21, 11711178. doi:10.1038/nbt874

[8] Lynn, D.M. (2006) Layers of opportunity: Nanostructured polymer assemblies for the delivery of macromolecular therapeutics. Soft Matter, 2, 269-273. doi:10.1039/b517860f

[9] Decher, G. (1997) Fuzzy nanoassemblies: Toward layered polymeric multicomposites. Science, 277, 1232-1237. doi:10.1126/science.277.5330.1232

[10] Jessel, N., Atalar, F., Lavalle, P., Mutterer, J., Decher, G., Schaaf, P., Voegel, J.-C. and Ogier, J. (2003) Bioactive coatings based on a polyelectrolyte multilayer architecture functionalized by embedded proteins. Advanced $\mathrm{Ma}$ terials, 15, 692-695. doi:10.1002/adma.200304634

[11] Benkirane-Jessel, N., Lavalle, P., Meyer, F., Audouin, F., Frisch, B., Schaaf, P., Ogier, J., Decher, G. and Voegel, J.-C. (2004) Control of monocyte morphology on and response to model surfaces for implants equipped with anti-inflammatory agents. Advanced Materials, 16, 15071511. doi:10.1002/adma.200306613

[12] Benkirane-Jessel, N., Schwinte, P., Falvey, P., Darcy, R., Haikel, Y., Schaaf, P., Voegel, J.-C. and Ogier, J. (2004) Build-up of polypeptide multilayer coatings with anti-inflammatory properties based on the embedding of piroxicam-cyclodextrin complexes. Advanced Functional Materials, 14, 174-182. doi:10.1002/adfm.200304413
[13] Jessel, N., Oulad-Abdeighani, M., Meyer, F., Lavalle, P., Haikel, Y., Schaaf, P. and Voegel, J.-C. (2006) Multiple and time-scheduled in situ DNA delivery mediated by beta-cyclodextrin embedded in a polyelectrolyte multilayer. Proceedings of the National Academy of Sciences, 103, 8618-8621. doi:10.1073/pnas.0508246103

[14] Kim, B.S., Park, S.W. and Hammond, P.T. (2008) Hydrogen-bonding layer-by-layer-assembled biodegradable polymeric micelles as drug delivery vehicles from surfaces. ACS Nano, 2, 386-392. doi:10.1021/nn700408z

[15] Benkirane-Jessel, N., Lavalle, P., Hubsch, E., Holl, V., Senger, B., Haikel, Y., Voegel, J.C., Ogier, J. and Schaaf, P. (2005) Short-time timing of the biological activity of functionalized polyelectrolyte multilayers. Advanced Functional Materials, 4, 648-654. doi:10.1002/adfm.200400129

[16] Dierich, A., Le Guen, E., Messaddeq, N., Stoltz, J.F., Netter, P., Schaaf, P., Voegel, J.-C. and Benkirane-Jessel, N. (2007) Bone formation mediated by synergy-acting growth factors embedded in a polyelectrolyte multilayer film. Advanced Materials, 19, 693-697. doi:10.1002/adma.200601271

[17] Facca, S., Cortez, C., Mendoza-Palomares, C., Messadeq, N., Dierich, A., Johnston, A.P., Mainard, D., Voegel, J.-C., Caruso, F. and Benkirane-Jessel, N. (2010) Active multilayered capsules for in vivo bone formation. Proceedings of the National Academy of Sciences, 107, 3406-3411. doi:10.1073/pnas.0908531107

[18] Krogman, K.C., Lowery, J.L., Zacharia, N.S., Rutledge, G.C. and Hammond, P.T. (2009) Spraying asymmetry into functional membranes layer-by-layer. Nature Materials, $\mathbf{8}$, 512-518. doi: $10.1038 /$ nmat 2430

[19] Nadiri, A., Kuchler-Bopp, S., Mjahed, H., Hu, B, Haikel, Y., Schaaf, P., Voegel, J.-C. and Benkirane-Jessel, N. (2007) Cell apoptosis control using BMP4 and noggin embedded in a polyelectrolyte multilayer film. Small, 9, 1577-1583. doi:10.1002/smll.200700115

[20] Mendoza-Palomares, C., Ferrand, A., Facca, S., Fioretti, F., Ladam, G., Kuchler-Bopp, S., Regnier, T., Mainard, D. and Benkirane-Jessel, N. (2012) Smart hybrid materials equipped by nanoreservoirs of therapeutics. ACS Nano, $\mathbf{6}$, 483-490. thoi:10.1021/nn203817t 